\title{
Inevitable Collision States for Motorcycle-to-Car Collision Scenarios
}

\author{
Giovanni Savino, Federico Giovannini, Michael Fitzharris, and Marco Pierini
}

\begin{abstract}
This paper presents a method to identify inevitable collision states (ICS) specifically for a motorcycle when interacting with an opponent passenger car in typical traffic scenarios. Previous ICS methods were applied to passenger cars or generic vehicles; however, the peculiarities of motorcycles urge the definition of specific methods for these vehicles. The findings extend the applicability of previous algorithms to include all motorcycle-tocar collisions, irrespective of collision configurations. ICS identification can be adopted as a triggering criterion for more invasive safety systems such as motorcycle autonomous emergency braking (MAEB), which require a last-resort approach in their initial phases of development. In this regard, this paper also presents an evaluation of an idealized MAEB through experiments simulating real-world crashes in a computer-based virtual environment.
\end{abstract}

Index Terms-Motorcycles, inevitable collision states, safety systems, autonomous emergency braking.

\section{INTRODUCTION}

$\mathbf{M}$ OTORCYCLES and mopeds are used worldwide and are particularly prominent in developing countries. Different patterns in fleets and motivations characterize their use in each specific context, although the risks for riders are typically much higher than for passenger cars [1], [2]. This is also reflected in the global number of over 180,000 estimated fatalities every year, in a proportion of $20 \%, 30 \%$ and $50 \%$ of passenger car users when considering respectively high, low and middle income countries [3].

Newly emergent active safety technologies can help reduce the number of severe casualties for motorcycle riders. These assistance systems actively intervene on the vehicle and its dynamics during conflict events. An example is anti-lock braking system (ABS) [4]. A further advance is autonomous emergency braking (AEB), currently under initial stages of development for motorcycle application. AEB is designed to automatically

Manuscript received June 15, 2014; revised July 17, 2015; accepted January 11, 2016. Date of publication February 18, 2016; date of current version August 25, 2016. This work was supported by the European Commission Seventh Framework Programme FP7/2007-2013 under Grant 328067 (ABRAM Project). The Associate Editor for this paper was A. Eskandarian.

G. Savino is with the Department of Industrial Engineering, University of Florence, 50121 Florence, Italy, and also with the Monash University Accident Research Center, Clayton, VIC 3168, Australia (e-mail: giovanni. savino@unifi.it).

F. Giovannini and M. Pierini are with the Department of Industrial Engineering, University of Florence, 50121 Florence, Italy.

M. Fitzharris is with the Monash University Accident Research Center, Clayton, VIC 3168, Australia.

Color versions of one or more of the figures in this paper are available online at http://ieeexplore.ieee.org.

Digital Object Identifier 10.1109/TITS.2016.2520084 apply the brakes or increase the braking action in the event of an imminent collision.

The natural aversion of riders towards assistive technologies [5] suggested the adoption of last resort solution approach for intrusive systems such as AEB: the system will be triggered only when an imminent conflict leads to inevitable collision, no matter what action the rider of the host vehicle nor the driver of the opponent vehicle try to implement.

Giovannini et al. addressed motorcycle maneuverability with field tests aiming at defining the basis to discriminate inevitable collisions [6]. The outcomes were applied in simple threat metrics for rear-end conflict scenarios [7]. For the purposes of AEB, the method to determine inevitable collision states (ICS) for a host motorcycle conflicting with an opponent passenger car needs to be extended to a wide range of motorcycle-to-car crash configurations.

Prior research explored the concept of state of inevitable collision providing model-based methods to identify ICS. However, the application of these ICS methods to powered two wheelers is not trivial. In fact, they should take account of the different characteristics of single-track vehicle dynamics, while allowing for real-time implementation.

In this paper, we defined and analyzed an ICS method for motorcycle vs. passenger car scenarios. The analysis revealed the characteristics of ICS for motorcycles, such as typical time to collision values (TTC) at ICS identification. We also presented a method for ICS check that can be implemented in a real-time, motorcycle-embedded system. Importantly, this method can be applied for intrusive, promising though, novel motorcycle safety technologies, such as autonomous emergency braking, which in their initial phases require a last-resort approach. An example of AEB for motorcycles was presented in our contribution. Finally, we performed an assessment of the method via detailed computer simulations reproducing a set of real-world crash situations.

\section{Previous Research}

\section{A. Literature Review on Inevitable Collision States}

Fraichard and Asama in 2004 firstly gave a definition of ICS and formalized its properties [8]. Martinez-Gomez and Fraichard started from the concept of ICS to develop an algorithm to check whether a generic planar robotic system is in ICS [9].

Kaempchen et al. in [10] proposed an algorithm searching for a combination of manoeuvres leading to a collision among a complete set of possible manoeuvres within the physical limits. 

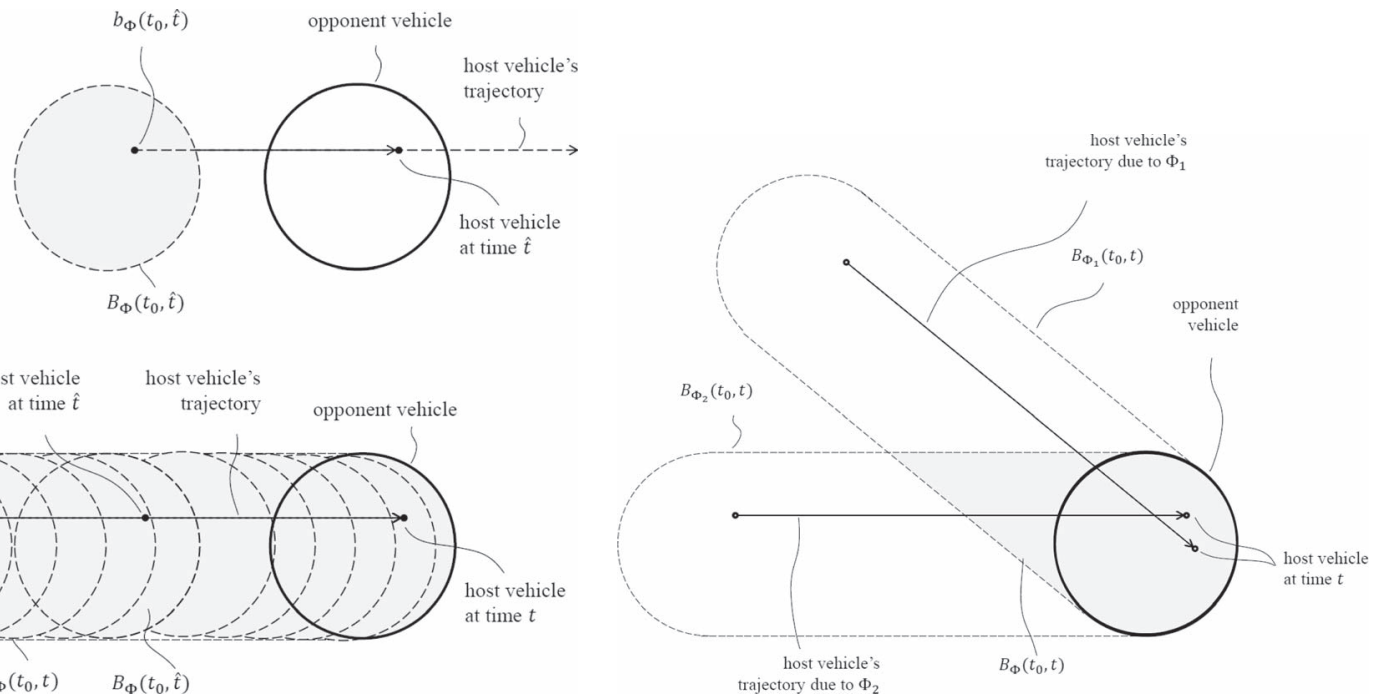

(a)

(b)

Fig. 1. (a) $\operatorname{ICS} b_{\phi}\left(t_{0}, \hat{t}\right), B_{\phi}\left(t_{0}, \hat{t}\right)$, and $B_{\phi}\left(t_{0}, t\right)$ for a host vehicle (punctual body) moving along a rectilinear trajectory defined by a maneuver $\phi$ and an opponent vehicle (extended body) in a fixed position. (b) ICS $B_{\phi_{1,2}}\left(t_{0}, t\right)$ for a host vehicle (punctual body) moving along rectilinear trajectories defined by the set of manoeuvres $\phi_{1}$ and $\phi_{2}$, and opponent vehicle (extended body) in a fixed position.

When the algorithm cannot find a solution, the collision is considered unavoidable. The algorithm was applied to passenger cars and modeled the vehicles as rectangles in the plane with a bicycle model. Although conservative, a limitation was that this algorithm did not consider several practical constraints for the manoeuvres, e.g. available power, and minimum steering radius. An alternative approach proposed by Eidehall and Petersson used Monte Carlo simulations instead of computing all the possible combinations of manoeuvres [11].

Different papers proposed applications of the concept of ICS to passenger cars (e.g. [12]), with purposes of safe route planning. When deterministic, the method turns out to be conservative with respect to crashes when ICS is over-estimated: all the states leading to inevitable collisions are avoided, together with possible non-ICS states.

A branch of studies analyzed this concept to define triggering algorithms for automatic emergency braking (AEB) for passenger cars. In the early stage of development of the AEB, the liability problem linked with its activation suggested the precautionary approach consisting in activating the system only when the collision becomes inevitable.

In this paper we present a novel application of established ICS principles to motorcycles, and in doing so, extend this work into modeling real-world motorcycle crashes.

\section{METhods}

\section{A. Inevitable Collision States}

Let us consider the ICS concept as defined in [8]. Given a generic state $s$ included in the state space $S$ of a system including a host vehicle and a set of static or dynamic obstacles, the inevitable collision states are a subset $B \subset S$ leading the host vehicle into a sure collision with at least one of the other vehicles for any action of the host and opponent vehicles, including extreme avoidance actions within physical limits.

\section{B. General Definitions}

For the definitions we referred to [8] adopting alternative notation. Let us denote the state $s$ (constituted by host vehicle plus opponent vehicles) at time $t_{0}$ included in the space $S$ and $\phi\left(t_{0}, \hat{t}\right)$ indicating a defined sequence of control inputs for the host vehicle and the opponent vehicle starting from time $t_{0}$ up to time $\hat{t}$.

A single ICS at time $t_{0}$ for a specific sequence of manoeuvres $\phi\left(t_{0}, \hat{t}\right)$, namely $b_{\phi}\left(t_{0}, \hat{t}\right)$ is an element of $S$ leading to a collision between host and opponent vehicles at time $\hat{t}$.

According to Property 2 of the ICS (Obstacle Union) [8], when considering extended objects, the ICS $B_{\phi}\left(t_{0}, \hat{t}\right)$ at time $t_{0}$ for the manoeuvres $\phi\left(t_{0}, \hat{t}\right)$ is the union of each and every $b_{\phi}\left(t_{0}, \hat{t}\right)$ (Fig. 1(a)).

$$
B_{\phi}\left(t_{0}, \hat{t}\right)=\bigcup b_{\phi}\left(t_{0}, \hat{t}\right) .
$$

For the same Property 2, the overall ICS for extended objects $B_{\phi}\left(t_{0}, t\right)$ for the specific manoeuvres $\phi\left(t_{0}, t\right)$ in the time horizon from $t_{0}$ to $t$ is the union of $B_{\phi}\left(t_{0}, \hat{t}\right)$, with $\hat{t}$ ranging from $t_{0}$ to $t$ (Fig. 1(a)).

$$
B_{\phi}=B_{\phi}\left(t_{0}, t\right)=\bigcup B_{\phi}\left(t_{0}, \hat{t}\right), \hat{t} \in\left[t_{0}, t\right] .
$$

According to Property 1 of the ICS (Control Inputs Intersection) [8], given a set $\Phi\left(t_{0}, t\right)$ of sequences of manoeuvres $\hat{\phi}\left(t_{0}, t\right)$, the ICS $B_{\Phi}\left(t_{0}, t\right)$ is the intersection of $B_{\hat{\phi}}\left(t_{0}, t\right)$ :

$$
B_{\Phi}=B_{\Phi}\left(t_{0}, t\right)=\bigcap B_{\hat{\phi}}\left(t_{0}, t\right), \quad \hat{\phi} \in \Phi .
$$

\section{Problem Statement}

Let us consider an on-road scenario with two vehicles about to collide: a host motorcycle and an opponent passenger car. The state $s$ at time $t_{0}$ is characterized by opponent car position, heading and velocity, and host motorcycle velocity. Previous 
work indicates that in multi-vehicle motorcycle crashes the host motorcycle in pre-cash phase is often traveling straight [13]. We then considered as initial state the host motorcycle traveling along a straight path in upright position. The lean angle at time $t_{0}$ was neglected, as supposed close to zero.

Both host motorcycle and opponent car are modeled as cuboids having their mass concentrated in their geometrical centers, respectively $C_{h v}$ and $C_{o v}$. Length and width of the cuboids are respectively $\left(L_{h v}, W_{h v}\right)$ for the host motorcycle and $\left(L_{o v}, W_{o v}\right)$ for the opponent car.

1) Opponent Car: The model for the tangential dynamics of the car we adopted for ICS purposes is similar to the model presented and validated in [14]. Considering fixed coordinates, the acceleration $\vec{a}$ of $C_{o v}$ and the angular rate $\omega$ of the vehicle are expressed by the following equations:

$$
\left\{\begin{array}{l}
\vec{a}=a_{T} u_{T} \vec{i}+a_{N} u_{N} \vec{j} \\
\omega=\frac{v}{\rho}
\end{array}\right.
$$

where $a_{T}$ and $a_{N}$ are the maximum tangential and normal accelerations, $u_{T}$ and $u_{N}$ tangential and normal controls, $\vec{i}$ and $\vec{j}$ tangential and normal unit vectors, $v$ current tangential velocity and $\rho=v^{2} /\left(a_{N}\left|u_{N}\right|\right)$ local radius of the path.

The following conditions apply:

$$
\begin{array}{ll}
a_{T}, a_{N} \in[0,1], & u_{T}, u_{N} \in[-1,1] \\
v \leq v_{\max }, & \rho>\rho_{\min }
\end{array}
$$

where $v_{\max }$ and $\rho_{\min }$ characterizing the vehicle represent the maximum velocity and the minimum turning radius, respectively. The sign of $u_{N}$ controls the direction of the curve. The parameter $a_{T}$ is computed as follows:

$$
a_{T}= \begin{cases}g, & u_{T} \leq 0 \vee 0<v \leq \frac{P}{g} \\ \frac{P}{v}, & u_{T}>0 \wedge v>\frac{P}{g} \\ 0, & v \leq 0\end{cases}
$$

where $g$ is the acceleration of gravity and $P$ is the power-tomass ratio characterizing the vehicle. Additionally, the tangential and normal accelerations are constrained according to the model of ellipse of adherence [15] as follows:

$$
\sqrt{\left(a_{T}\left|u_{N}\right|\right)^{2}+\left(a_{N}\left|u_{T}\right|\right)^{2}} \leq g^{2} .
$$

2) Motorcycle Model: The motorcycle model is in charge of reproducing realistic performances of extreme avoidance manoeuvres in terms of space. In other words, the model must mimic the actual margin to avoid a collision when using typical emergency controls, namely pure braking, pure swerving and a combination of braking and swerving.

When tuned according to the upper limit capabilities of the motorcycle, the model will result conservative in the description of the collision avoidance performances, thus avoiding false positive cases. Conversely, if the actual avoidance capabilities of the motorcycle were far below the limits indicated by the model, ICS would be over duly under-estimated, thus decrementing the potential for possible intervention at ICS detection, both time- and space-wise.
Details regarding high performance braking maneuver along a straight are given in [15]. In [16] the authors proposed a model of braking where the upper limit for the emergency braking deceleration $d_{\max }$ is a function of the available roadtire adherence $\mu$, the motorcycle mass distribution $a / h$ being the ratio between longitudinal position and height of $C_{h v}$, the maximum braking force $F_{\max }$ produced by the braking system, and the aerodynamic resistance as a function of velocity and drag coefficient $D_{T}$

$$
d_{\max }=f\left(\mu, \frac{a}{h}, F_{\max }, v, D_{T}\right) .
$$

Assuming that the rider begins an optimal braking maneuver at time $t_{0}$, a full deceleration is achievable after a time interval of $\Delta t_{b}$ due to the unsteady phase in the braking system (typically not shorter than $0.3 \mathrm{~s}$ for any motorcycle [17]). A linear increase of deceleration from zero to $d_{\max }$ during $\Delta t_{b}$ was assumed as optimistic and therefore conservative with respect to actual performances.

A novelty of the present study is to incorporate realistic lateral avoidance performances of motorcycles into the ICS computation. These performances were investigated by the authors in a previous study [6]. In that study, the last second swerve maneuver was modeled considering an instantaneous change from a steady state with zero curvature to a steady state at constant curvature $C_{\max }$ with constant velocity $v_{h v}$, where

$$
C_{\max }=\frac{g \tan \left(\varphi_{\max }\right)}{v_{h v}^{2}} .
$$

The parameter $\varphi_{\max }$ is the maximum lean angle achievable during the last second swerving (due to the constraints of vehicle geometry and adherence). The last second swerve model is supported by the results of a series of tests with volunteer riders riding a scooter towards a fixed obstacle in a controlled environment [6].

For the purposed of the present study, the extreme braking and swerving manoeuvres for the motorcycle outlined in the previous paragraphs were described using (4). In particular, the lateral dynamics of (4) and the last second swerve model overlap when assuming $\rho=1 / C_{\max }$.

Let us now consider a combined action of longitudinal and lateral controls. Kaempchen et al. in [10] used a dynamic model for passenger car similar to the one adopted in the present paper and showed that specific combination of braking and swerving the vehicle model may produce higher avoidance performances than a pure action. The combination of lateral and longitudinal control for the motorcycle was simplified in accordance with the Kamm's circle model stating that the vectorial sum of the longitudinal and lateral friction coefficients cannot exceed $\mu$ [18]. Therefore when the braking action produces a deceleration $d^{*}$ (with upper limit $\mu g$ ) and vehicle geometry guarantees clearance with the ground for a maximum lean angle $\varphi^{*}$, the lateral avoidance maneuver was modeled using (9) with:

$$
\varphi_{\max }=\min \left\{\varphi^{*}, \arctan \left(\frac{\sqrt{\mu^{2} g^{2}-d^{* 2}}}{g}\right)\right\} .
$$




\section{Proposed Solution}

Considering the system constituted by host motorcycle and one single opponent car at current time $t_{0}$, the state $s\left(t_{0}\right)$ of the system is an element of a penta-dimensional space $S$, defined as:

$$
s\left(t_{0}\right)=\left\{x_{o v}\left(t_{0}\right), y_{o v}\left(t_{0}\right), v_{h v}\left(t_{0}\right), v_{o v}\left(t_{0}\right), \theta_{o v}\left(t_{0}\right)\right\}
$$

where $x_{o v}$ and $y_{o v}$ are the Cartesian coordinates of the geometrical centre of the opponent car with respect to the host motorcycle, $v_{h v}$ and $v_{o v}$ are respectively the velocity of the host and opponent car in a fixed reference system centered in $C_{h v}$, and $\theta_{o v}$ is the relative heading of the opponent car, all the variables considered at time $t_{0}$.

To determine the ICS $B \subset S$, a finite set of manoeuvres $\hat{\Phi}$ is analyzed in a $2 \mathrm{D}$-slice of the state domain. This approach is based on the assumption that a sufficiently ample and well chosen set of manoeuvres $\hat{\Phi}$ practically covers all the collision avoidance possibilities achievable with the infinite set $\Phi$ of feasible manoeuvres within the physical limits of the considered vehicles. The assumption is stated as follows:

$$
B(\hat{\Phi}) \approx B(\Phi) .
$$

The solving method took inspiration from the ICS checker proposed by Martinez-Gomez and Fraichard [9]. The problem of identifying $B$ is reduced to the problem of computing the region $A$ of coordinates $\left(x_{o v}, y_{o v}\right) \in A$ that satisfy the ICS condition in the state slice $R$ defined as follows:

$$
\left.R \equiv S\right|_{v_{h v}\left(t_{0}\right), v_{o v}\left(t_{0}\right), \theta_{o v}\left(t_{0}\right)}, R \subset \mathbb{R}^{2} .
$$

In other words, the identification of ICS for a given current state $s\left(t_{0}\right)$ as in (11) is reduced to check whether the position of the opponent car at time $t_{0}$ is in the region $A\left(t_{0}\right)$.

The next steps consist in the definition of the time horizon $t$ and the set of manoeuvres $\hat{\Phi}$. A limited time horizon rather than the infinite one required by the ICS theory can be chosen noticing that due to the characteristics of the host motorcycle and opponent car in terms of maneuverability and dimensions, foreseen inevitable collisions always take place within a limited time frame from the observation time $t_{0}$ (typically less than $1 \mathrm{~s}$ ). Concerning the manoeuvres $\hat{\Phi}$, a representative set was selected using a heuristic approach as described hereafter. In the 2Dslice space $R$, a set of sample states were identified considering a combination of the three state variables in their minimum, average and maximum values: a total of 27 sample states were identified. A basic maneuver set $\Phi_{\text {ref }}$ and additional sets of increasing number of manoeuvres were defined. The selected manoeuvres were constant in time, according to the models of driver manoeuvres presented in [19], when neglecting initial ramps to be conservative. For each state element the corresponding ICS $B_{\Phi^{*}}$ was computed, being $\Phi^{*}$ the tested maneuver set. As the number of manoeuvres increases, the area of $\mathrm{B}$ reduces due to Property 1 . The final set of manoeuvres (Table I) was chosen in order to guarantee the minimum average area of $B$ for the sample states, assuming that a different or larger set of manoeuvres would not affect $B$, according to assumption (12). An exemplification of the effects of larger sets of manoeuvres on the average of $B$ is given in Fig. 2.
TABLE I

REFERENCE MANOEUVRES

\begin{tabular}{lrrrr}
\hline \hline & \multicolumn{2}{c}{$\mathrm{HM}$} & \multicolumn{2}{c}{ OV } \\
N. & $u_{T}$ & $u_{N}$ & $u_{T}$ & $u_{N}$ \\
\hline 1 & -1 & 0 & -1 & 0 \\
2 & -1 & 0 & 0 & -1 \\
3 & -1 & 0 & 0 & 1 \\
4 & 0 & 1 & -1 & 0 \\
5 & 0 & -1 & -1 & 0 \\
6 & 0 & 1 & 0 & 1 \\
7 & 0 & -1 & 0 & -1 \\
8 & -0.5 & -1 & -0.5 & -1 \\
9 & -0.5 & 1 & -0.5 & 1 \\
10 & 0.5 & 1 & -0.5 & 1 \\
11 & 0.5 & -1 & -0.5 & -1 \\
12 & -0.5 & 1 & 0.5 & 1 \\
13 & -0.5 & -1 & 0.5 & -1 \\
14 & 0.5 & -1 & -0.5 & 1 \\
15 & 0.5 & 1 & -0.5 & -1 \\
16 & -0.5 & -1 & 0.5 & 1 \\
17 & -0.5 & 1 & 0.5 & -1 \\
\hline \hline
\end{tabular}

HM host motorcycle

OV opponent vehicle

$u_{T}$ tangential control

$u_{N}$ normal control

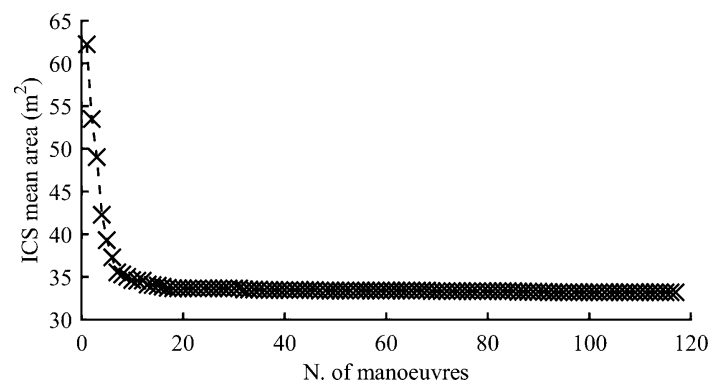

Fig. 2. Mean areas over 27 sample states for ICS regions obtained using different sets of reference manoeuvres. The first set of manoeuvres are detailed in Table I. The control values for the second set of manoeuvres were randomly generated with uniform distribution; $u_{T} \in[-1,1], u_{N} \in\{-1,0,1\}$.

\section{E. Look Up Table}

The method described in the previous paragraphs can be applied in real time as follows. At every time step, an obstacle detection unit on board the host motorcycle detects the opponent car's position, velocity and heading. An algorithm computes the area $A$ corresponding to ICS for the current 2Dslice state $R$. Eventually, a comparison between the area $A$ and the actual position of the opponent car establishes whether the vehicles are in an ICS or not.

In contrast to on-line computation, we present an alternative way to apply ICS based on a look up table created off-line.

In order to maintain a conservative approach notwithstanding the discretization produced by the look up table, for each input except for the vehicle velocities both the upper and lower approximating values are considered. Thus the method requires 8 accesses to the look up table each time step.

The look up table is particularly useful for a global analysis of the performances of the ICS. The steps for the creation of the look up table are described hereafter.

1) The characteristics of the host motorcycle and opponent car in terms of dimensions and maneuverability are determined a priori. To be conservative, lower limits for vehicles dimensions and upper limits for vehicles maneuverability ought to be chosen. 
TABLE II

EXAMPLE of Discretization TABLE for the State VARiables

\begin{tabular}{lcccc}
\hline Variable name & Symbol & Unit & Range & Step \\
\hline HM velocity & $v_{h v}$ & $\mathrm{~m} / \mathrm{s}$ & $0-36$ & 3 \\
OC velocity & $v_{o v}$ & $\mathrm{~m} / \mathrm{s}$ & $0-36$ & 3 \\
OC heading & $\theta_{o v}$ & $\mathrm{rad}$ & $0-\pi^{*}$ & $\pi / 36$ \\
OC longitudinal position & $x_{o v}$ & $\mathrm{~m}$ & $0-40$ & 0.2 \\
OC lateral position & $y_{o v}$ & $\mathrm{~m}$ & $-20-20$ & 0.2 \\
\hline
\end{tabular}

HM: host motorcycle; OC: opponent car.

This look up table would result in 207.36 million elements

corresponding to a minimum memory usage without compression of 25.92 MBytes.

*The table is symmetric with respect to the opponent car heading.

TABLE III

PARAMETERS USED TO COMPUTE ICS

\begin{tabular}{lccc}
\hline \hline Variable name & Symbol & Unit & Value \\
\hline Max. adherence & $\mu$ & & 1.0 \\
HM max. swerve lean angle & $\varphi_{\max }$ & $\mathrm{rad}$ & 0.61 \\
OV max. lateral acceleration & & $\mathrm{m} / \mathrm{s}^{2}$ & 7.0 \\
HM min. radius & $\rho_{h \operatorname{mmin}}$ & $\mathrm{m}$ & 4.0 \\
OC min. radius & $\rho_{\text {ovmin }}$ & $\mathrm{m}$ & 4.0 \\
HM max. velocity & $v_{h \max }$ & $\mathrm{m} / \mathrm{s}$ & 50 \\
OC max. velocity & $v_{\text {ocmax }}$ & $\mathrm{m} / \mathrm{s}$ & 50 \\
HM max. specific power & $P_{h \operatorname{mmax}}$ & $\mathrm{kW} / \mathrm{ton}$ & 80 \\
OC max. specific power & $P_{o c \max }$ & $\mathrm{kW} / \mathrm{ton}$ & 50 \\
HM delay before full braking & $\Delta t_{f b}$ & $\mathrm{~s}$ & 0.2 \\
HM length, width & $l_{h m}, w_{h m}$ & $\mathrm{~m}$ & $2.0,1.0$ \\
OC length, width & $l_{O c}, w_{o c}$ & $\mathrm{~m}$ & $4.0,2.0$ \\
Time horizon for $I C S$ & $\mathrm{t}$ & $\mathrm{s}$ & 1.0 \\
\hline \hline
\end{tabular}

HM: host motorcycle; OC: opponent car.

2) The three-dimensional state space $R$ consisting of host motorcycle velocity and opponent car velocity $\&$ heading is defined in their range and discretized. Similarly, the rectangular field $T$ in front of the motorcycle is defined in range and discretized into a grid.

3) For each discrete state $r \in R$, the area $A$ of inevitable collision is computed and discretized over $T$.

4) A five-dimensional look up table is built combining the discretized space $R$ and the discretized field $T$. Each element of the look up table identifies a discretized state $s$. Each value stored in the look up table is a Boolean value indicating whether the corresponding discrete state $s$ is an ICS $B$ or not.

An example of discretization and a preview of the look up table dimension is provided in Table II.

\section{F. Analysis of the ICS Matrix}

As an example, the proposed method was applied to analyze the ICS for a motorcycle and a passenger car using the parameters given in Table III and the set of manoeuvres given in Table I. The result was a look up table identifying the ICS between the two given vehicles. From here on this look up table will be addressed as ICS matrix. The first analysis considered the simple configuration of motorcycle approaching a fixed obstacle and compared the current method with the results of the methods proposed in [6] [Fig. 3(a)]. In car-following scenarios, the authors in [16] proposed to consider the moving

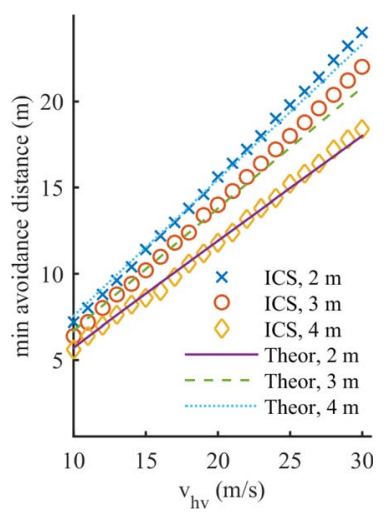

(a)

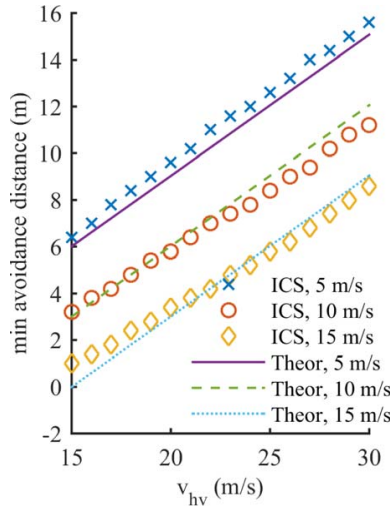

(b)
Fig. 3. (a) Minimum swerving distances for a fixed, rectangular obstacle (obstacle width equal to $2 \mathrm{~m}, 3 \mathrm{~m}$, and $4 \mathrm{~m}$ ) computed with the ICS method and the theoretical formula proposed by [6] (labeled as Theor. in the plot). (b) Minimum swerving distances for a moving, rectangular obstacle (obstacle velocity equal to $5 \mathrm{~m} / \mathrm{s}, 10 \mathrm{~m} / \mathrm{s}$, and $15 \mathrm{~m} / \mathrm{s}$ ) computed with the ICS method and the formula proposed by [6] (labeled as Theor. in the plot).

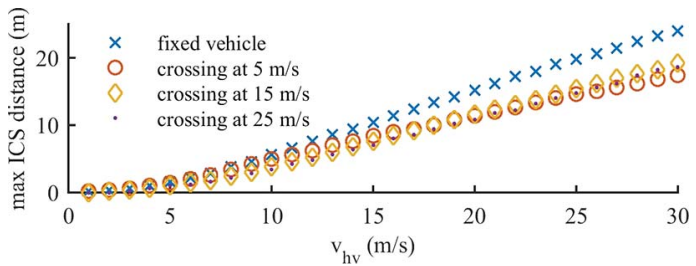

Fig. 4. Max distance for detecting an ICS state considering an opposing passenger car in fixed position and travelling $5 \mathrm{~m} / \mathrm{s}, 15 \mathrm{~m} / \mathrm{s}$, and $25 \mathrm{~m} / \mathrm{s}$ across the host motorcycle's path.

car as a fixed obstacle with the motorcycle traveling at adjusted velocity $v_{\text {rel }}$ equal to the approaching velocity:

$$
\vec{v}_{\text {rel }}=\vec{v}_{h v}-\vec{v}_{o v} .
$$

A comparison with the current ICS matrix is provided in Fig. 3(b).

For crash scenarios with an opponent car crossing the host motorcycle path, in [7] the authors proposed to consider the slowly crossing car as a fixed obstacle. In Fig. 4 a comparison between fixed obstacle scenario and crossing passenger car scenario is provided using the current ICS matrix. The analysis showed that at low velocities of the host motorcycles the velocity of the opponent car scarcely affects the distance at which the ICS is obtained. In addition, at high velocities of the host motorcycle the ICS is obtained at shorter distance when the opponent car is moving. These differences are due to the possibility for the opponent car to maneuver.

Further analysis of the ICS matrix evaluated the time to collision at ICS detection (TTC). The analysis was conducted considering the TTC for each 2D-slice belonging to $R$. The maximum TTC in each 2D-slice refers to one single approaching direction of the opponent car with respect to the host motorcycle. A more significant metric should reflect typical values of TTC when ICS is firstly detected. Let us consider an ICS area $A$ for a specific state $r \in R$. The direction of the relative velocity $-\vec{v}_{\text {rel }}$ identifies the portion $\partial^{\prime} A$ of the edge $\partial A$ from which the 


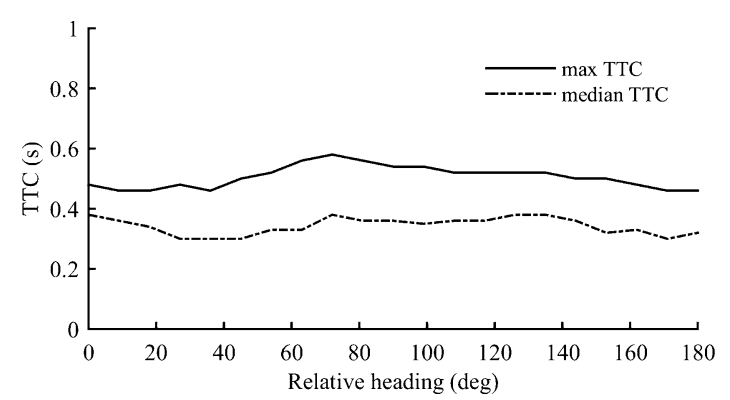

Fig. 5. Comparison between maximum and median TTC values computed on the approaching edge of $\partial^{\prime} A$, for an opponent car traveling at $5 \mathrm{~m} / \mathrm{s}$ with relative heading in the range $0^{\circ}-180^{\circ}$ when the host motorcycle is traveling at $20 \mathrm{~m} / \mathrm{s}$.

opponent car will enter. $\overline{\text { TTC }}$ is the median value of TTC on the edge $\partial^{\prime} A$ projected onto the direction $\vec{n}$ orthogonal to $\vec{v}_{\text {rel }}$. A comparison between maximum and median values of the time to collision for the ICS matrix is given in Fig. 5. The TTC at ICS detection is close to the maximum value when the projection of the vehicles trajectories would result in a collision of the host motorcycle in the centre of the opponent car, whereas TTC is close to zero when the projection of the trajectories would produce a sideswipe. A further example of typical median TTC values is given in Fig. 6(a) and (b) showing the influence of the vehicles velocities and relative heading.

\section{Demonstration OF the Application OF ICS TO REAL WORLD PROBLEMS}

The applicability of the proposed ICS identification method encompasses the fields of active and passive safety countermeasures. In this paper, an exemplification of the ICS matrix applied to a promising active countermeasure-motorcycle autonomous emergency braking (MAEB) — is given.

\section{A. MAEB System}

Autonomous emergency braking (AEB) was designed to slow down the host vehicle in the case the system detected an imminent collision, with documented impact in reducing the number of passenger car crashes [20].

The applicability of AEB to motorcycles was initially evaluated by two independent studies [17], [21]. One of those concepts was further developed to build up a prototype for on road testing [16]. This specific system was taken as baseline to exemplify the applicability of ICS to a feasible motorcycle safety technology.

The system included an obstacle detection system and a state sensing unit to collect the inputs for ICS assessment. In extension to MAEB prototype, the present paper considered the system to operate in series with a two-channel anti-lock braking system, as recommended in [16].

The logic to activate autonomous braking consisted in the three following conditions: a) an ICS has been detected; b) the rider is not acting on the brake controls; and c) the motorcycle is in upright position (lean angle $<10^{\circ}$ ). Autonomous braking consisted in a deceleration of the motorcycle with target value

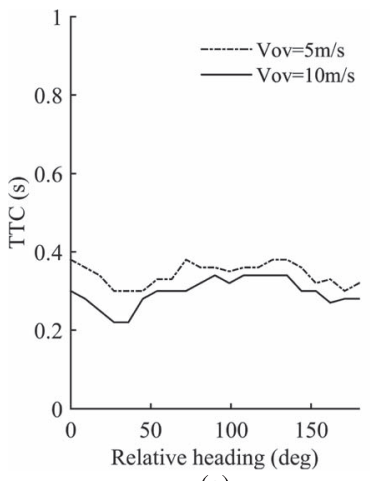

(a)

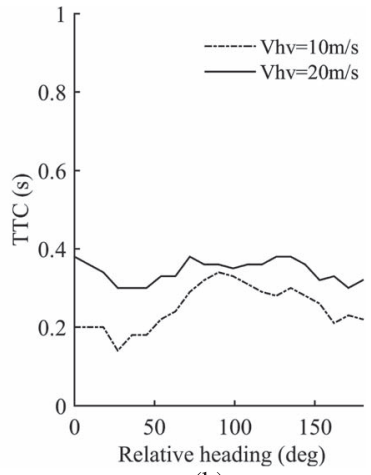

(b)
Fig. 6. Median time to collision TTC at ICS detection with relative heading in the range $0^{\circ}-180^{\circ}$, when: (a) the host motorcycle is traveling at $20 \mathrm{~m} / \mathrm{s}$; (b) the opponent car is traveling at $5 \mathrm{~m} / \mathrm{s}$.

of $3 \mathrm{~m} / \mathrm{s}^{2}$. This automatic maneuver was proved to be feasible [16], [22], [23]. Additional details about the reference MAEB system are given in [24].

\section{B. Simulations}

An assessment of the proposed method to determine ICS in generic pre-crash configuration was performed via crash reconstructions in virtual environment.

1) Method: The method used for the evaluation through simulations was inspired by [7] focusing on the evaluation of MAEB performances in fatal rear end crashes using a simple triggering algorithm. In the present paper, a set of real world, in-depth investigated crashes were recreated in a computerbased virtual environment. Each case consisted in a passenger car crossing the path of the host motorcycle resulting in a collision of the motorcycle into the car. For each case, an inscale planar representation of the real road branches was created with the software PreScan (TASS International, Helmond, The Netherlands) [25]. The road-tire adherence coefficient was set to 0.85 , as in all the crash cases the road surface was reported to be dry and in fair conditions. In each case the dimensions of the virtual vehicles were set to mimic the dimensions of the actual vehicles involved in the crash. The passenger car was modeled as an object moving along a predetermined path in order to mimic the actual behavior of the vehicle. In contrast, a realistic dynamic behavior of the motorcycle model was obtained using a detailed multi body model generated by the software tool Bikesim (Mechanical Simulation, Ann Arbor, MI) coupled with PreScan in a Matlab Simulink environment (MathWorks, Natick, MA).

The specifications of the disc brakes adopted in the simulations are shown in Table IV. The hydraulic pressure on the front brake master cylinder was controlled in open loop to produce the target deceleration of $0.3 \mathrm{~g}$.

A preliminary activity analyzed the behavior of MAEB in the simulation environment in a set of basic crash configurations. Ten simulations were generated considering a combination of two sets of velocities $(5 \mathrm{~m} / \mathrm{s}$ and $10 \mathrm{~m} / \mathrm{s} ; 10 \mathrm{~m} / \mathrm{s}$ and $20 \mathrm{~m} / \mathrm{s}$ for the host motorcycle and opponent car, respectively) each one using five different relative heading values $\left(0^{\circ}, 45^{\circ}, 90^{\circ}, 135^{\circ}\right.$, 
TABLE IV

BRAKING SYSTEM SPECIFICATIONS

\begin{tabular}{llc}
\hline \hline Type & Front [CPA, DER, PFC ] & Rear [CPA, DER, PFC] \\
\hline Moped & $1962 \mathrm{~mm}^{2}, 140 \mathrm{~mm}, 0.40$ & $1962 \mathrm{~mm}^{2}, 110 \mathrm{~mm}, 0.40$ \\
Sports & $3215 \mathrm{~mm}^{2}, 145 \mathrm{~mm}, 0.45$ & $1607 \mathrm{~mm}^{2}, 100 \mathrm{~mm}, 0.45$ \\
Touring & $3215 \mathrm{~mm}^{2}, 150 \mathrm{~mm}, 0.45$ & $1607 \mathrm{~mm}^{2}, 130 \mathrm{~mm}, 0.45$ \\
\hline CPA: caliper piston area. DER: Disc effective radius. PFC: pad friction \\
coefficient
\end{tabular}

$\left.180^{\circ}\right)$. In these simulations the velocity of the vehicles was constant and the vehicles were traveling along straight paths. The results were then compared with the theoretical values of TTC provided in Section III-F, showing an agreement between theoretical and simulated data (Fig. 7).

This preliminary analysis was followed by the simulations of the real world crash cases. For each simulated case, the initial state of the host motorcycle and opponent passenger car were set according to the case report available from the in-depth investigation. The virtual cases were then tuned by running the simulations in the reference configuration (MAEB not active) using a trial-and-error method to obtain an outcome compatible with the crash reports in terms of: i) point of collision; ii) vehicle orientations; iii) impact speed.

At the end of the tuning process, each scenario was replicated and modified to obtain two near miss cases by: a) slightly varying the initial relative position of the vehicles; $b$ ) introducing a last second swerve maneuver operated by the virtual rider.

The last second swerving manoeuvres were modeled using a closed loop control on the motorcycle lean angle. The steering torque $\tau_{s t}$ was determined as a function of actual and reference lean angle values, according to the following equation:

$\tau_{s t}=-k_{p}\left(\varphi_{\mathrm{ref}}-\varphi_{\mathrm{act}}\right)-k_{i} \int\left(\varphi_{\mathrm{ref}}-\varphi_{\mathrm{act}}\right) d t+k_{i} \dot{\varphi}_{\mathrm{act}}$

where $k_{p}, k_{i}$, and $k_{d}$ are the proportional, integral and derivative gains of the controllers. The gains are typically function of the actual velocity [26]. The reference lean angle during the swerving maneuver was modeled as a sine curve with an amplitude of $30^{\circ}$ and $35^{\circ}$ of amplitude for the scooters and for the other motorcycle types respectively, and $0.66 \mathrm{~Hz}$ of frequency. The shape of the input signal aimed to simulate a complete swerving maneuver, composed of a steering and a counter-steering phase [6].

The aim of the modified configuration cases was to confirm that the ICS recognition did not produce false triggering events (i.e. ICS detection for a near miss collision).

Finally, six simulations were run for each crash case: two simulations in the actual configuration with MAEB disabled (reference) and MAEB active; and four simulations in the modified configurations: near miss type (a) and (b), each one with MAEB disabled and active.

2) Material: The real-world crash cases used in the demonstration of the ICS applicability were selected from the dataset of the project Powered two wheeler Integrated Safety (PISa, 6th Framework Programme of the European Commission). Details about the PISa dataset are given elsewhere [24], [27].

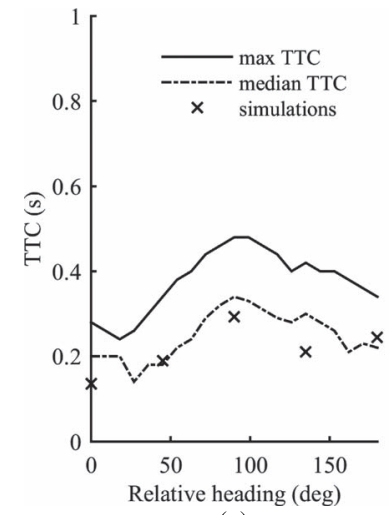

(a)

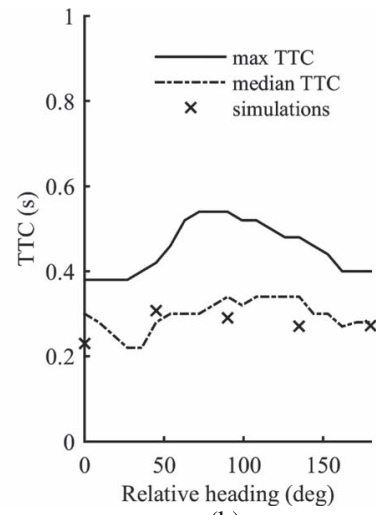

(b)
Fig. 7. Results from the validation of the simulation environment considering two combinations of constant velocity and a set of relative heading. The time to collision TTC of the simulations is plotted over the minimum, median and maximum TTC on the approaching edge of $\partial^{\prime} A$ extracted from the ICS matrix. (a) $v_{\mathrm{HM}}=10 \mathrm{~m} / \mathrm{s}, v_{\mathrm{OC}}=5 \mathrm{~m} / \mathrm{s}$ (b) $v_{\mathrm{HM}}=20 \mathrm{~m} / \mathrm{s}, v_{\mathrm{OC}}=10 \mathrm{~m} / \mathrm{s}$. HM: host motorcycle; OC: opponent car.

A subset of cases was shortlisted from the PISa dataset for detailed simulations according to the following three criteria:

- the rider did not actuate brake controls prior to crash;

- the collision took place at intersection;

- the collision involved one motorcycle and one passenger car.

The final set of crashes consisted in the 10 cases summarized in Table V (see Appendix for a description of each crash case).

3) Results: When considering the real crash cases, the proposed method for ICS identification did not produce any false triggering event in the first type of near miss cases (those obtained varying the initial relative positions of host and opponent vehicle). Concerning the second type of near misses (involving a last second swerve), false ICS was detected in two cases during the initial phase. However, in these cases ICS triggering occurred after initiating the swerve maneuver $(0.54 \mathrm{~s}$ and $0.50 \mathrm{~s}$ respectively), thus violating one of the assumptions of the proposed method which considers the motorcycle travelling along a straight path. An actual false triggering would be avoided with a simple state checker for the motorcycle discriminating whether a lateral movement has started or not. A possible algorithm was presented and validated in [29].

The results of the simulations are summarized with the parameters time to collision (TTC) from ICS detection to crash (MAEB not active) and impact speed reduction (ISR) produced by MAEB, i.e. the difference between the impact speed of the host motorcycle in actual conditions and when MAEB is active (Table V). A typical example of intervention of MAEB during the simulations is shown in Fig. 8 (see Fig. 10 for the other simulated cases).

The maximum TTC obtained in the simulated cases was $0.33 \mathrm{~s}$ in case 4 . In two of the simulated cases the ICS was detected in proximity of the actual collision time (cases 2 and 7). In three cases the ISR produced by MAEB was negligible (cases 1,2, and 7), whereas the maximum impact speed reduction obtained in the simulations was $0.85 \mathrm{~m} / \mathrm{s}$ (case 4). In Fig. 9 a synthesis of the results is given. 
TABLE V

SYNTHETIC CHART OF SAMPLE CRASHES

\begin{tabular}{lllllcccc}
\hline \hline Case & HM trajectory & OC manoeuvre & DCA $(*)$ & HM type & HM is $(\mathrm{m} / \mathrm{s})$ & Validation $(* *)$ & TTC $(\mathrm{s})$ & ISR $(\mathrm{m} / \mathrm{s})$ \\
\hline 1 & Lane change & Right turn & UK 147 & Moped & 13.23 & - & 0.16 & 0.02 \\
2 & Straight & Right turn & UK 147 & Moped & 8.85 & - & 0.03 \\
3 & Straight & Right turn & UK 121 & Touring & 13.95 & - & 0.32 \\
4 & Lane change & Right turn & UK 152 & Sports & 13.35 & - & 0.33 & 0.27 \\
5 & Straight & Right turn & DE 152 & Touring & 19.32 & - & 0.27 & 0.04 \\
6 & Lane change & Right turn & UK 152 & Touring & 24.29 & - & 0.71 \\
7 & Straight & Straight & UK 110 & Sports & 8.84 & -20 & 0.61 \\
8 & Straight & Right turn & UK 147 & Moped & 8.84 & - & 0.21 \\
9 & Straight & Straight & UK 110 & Touring & 19.91 & - & 0.28 \\
10 & Straight & Right turn & UK 147 & Touring & 9.46 & - & 0.44 \\
\hline \hline
\end{tabular}

HM: host motorcycle; OC: opponent car. (*) Country and DCA code. DCA represents the definitions for classifying accidents proposed by VicRoads [28]. (**) False triggering events for the near misses cases. A: crash avoided by translating the initial position of the host motorcycle. $L$ : crash avoided by last second swerve manoeuvre.
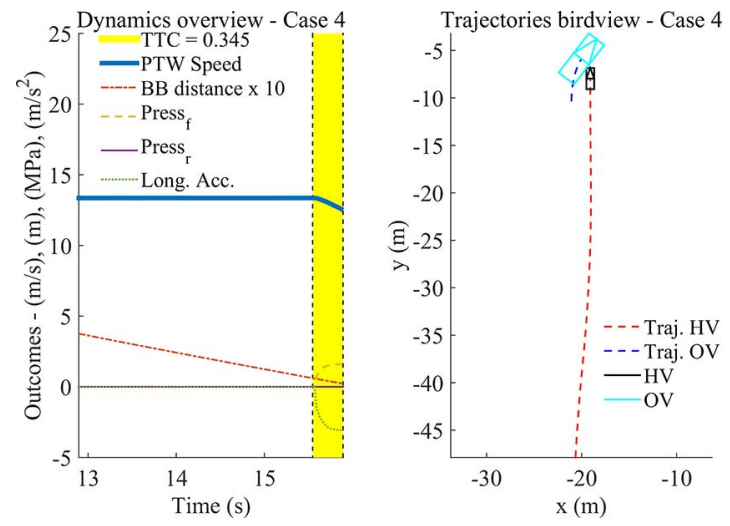

Fig. 8. Signal plot and trajectory plot of simulated case 4 with MAEB intervention.

\section{DISCUSSION}

The contribution of the present paper is to extend the implementations of the theory of ICS to a host motorcycle based on a characterization of the actual avoidance capabilities of such vehicles. The paper showed that the proposed method for ICS produced similar results of previous algorithms in basic precrash scenarios, but also extended the applicability to a large variety of motorcycle-to-car crash configurations, in which a simplistic algorithm may over-estimate the TTC value at which the collision becomes inevitable.

The foreseen impact of this contribution is twofold. First, it enables the application to motorcycles of more intrusive, promising though, safety systems such as AEB or airbags. In fact, the typical aversion of motorcycle riders towards assistive technologies can be addressed by designing a safety system that deploys only after the collision has become physically inevitable. It also enables preliminary evaluations of these technologies thanks to the analysis of the time to collision values. Secondly, this contribution can be used to enhance safety systems designed to avoid or mitigate collisions between motorcycles and passenger cars, such as collaborative AEB systems. In fact, the knowledge whether an inevitable collision state has or has not been reached can make car AEB deployment more robust, and collaborative deployment strategies are enabled as well. An example of these approaches was presented by the authors [30].

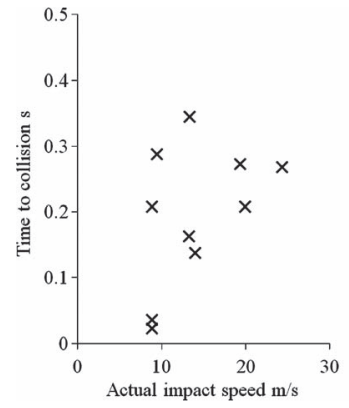

(a)

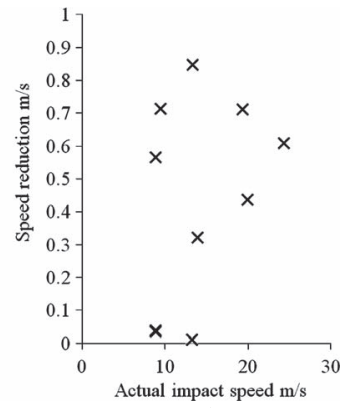

(b)
Fig. 9. Results from the simulations of the 10 crash cases: (a) Time to collision vs. actual impact speed. (b) Impact speed reduction vs. actual impact speed.

\section{A. Limitations}

The proposed method for the identification of ICS between a host motorcycle and an opponent car requires that the host motorcycle is traveling straight and has not started a lateral avoidance maneuver. Notwithstanding this limitation, the method is expected to apply to a relevant proportion of threatening situations of multi-vehicular crashes involving motorcycles [27].

The method computed the ICS considering one single opponent car. In case of multiple obstacles the approach is conservative. In fact, as Fraichard demonstrated in the previously cited work [8], in case of multiple obstacles the ICS area is the same or larger that the union of the single ICS areas computed for each obstacle at a time. Further developments may allow to increase the TTC values for imminent crash situations involving multiple obstacles.

The analysis of the ICS matrix showed that the minimum TTC value becomes small in a range of relative headings for the opponent car. In other words, in particular pre-crash configurations in which the opponent car enters the ICS area in the point of a minimum TTC value the ICS matrix cannot anticipate the collision since a combination of avoidance maneuvers may still avoid the collision. Nonetheless, median TTC values have limited variations in the whole range of relative headings.

Concerning the demonstration of ICS application to MAEB, the simulations indicated the feasibility of the method for real world crashes. Further, the simulations showed the feasibility of a lookup table approach and provided quantitative estimation of the potential effects of such system in terms of impact speed reduction (ISR). 

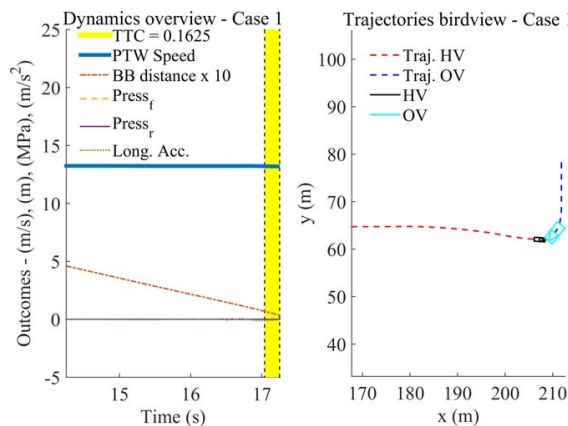

(a)
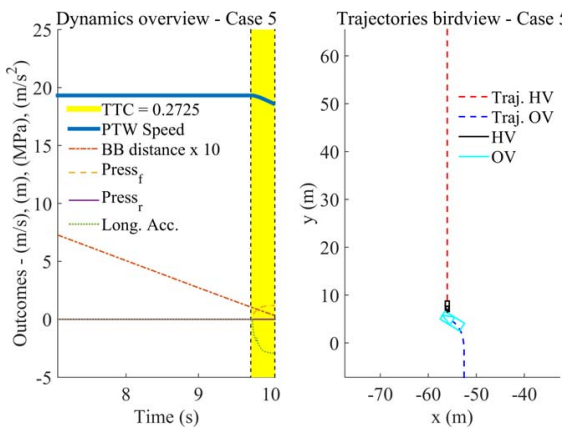

(d)
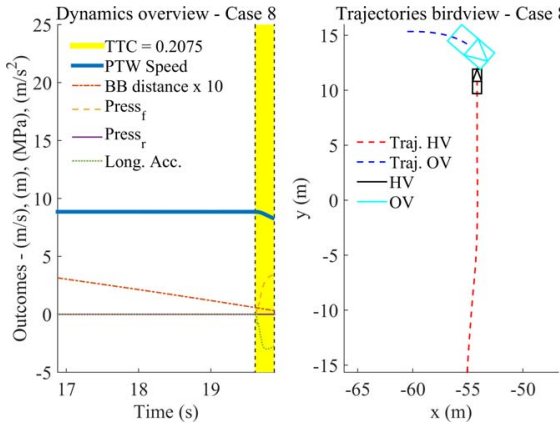

(g)
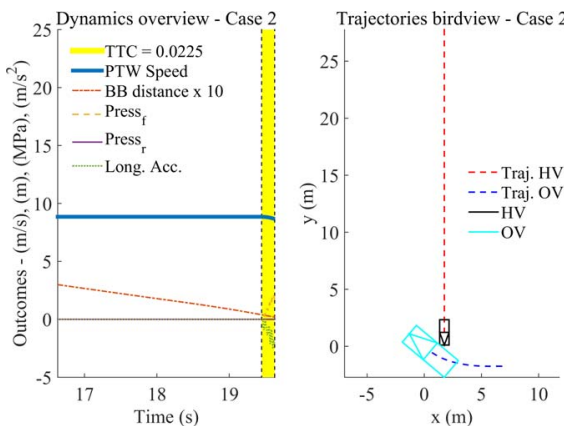

(b)
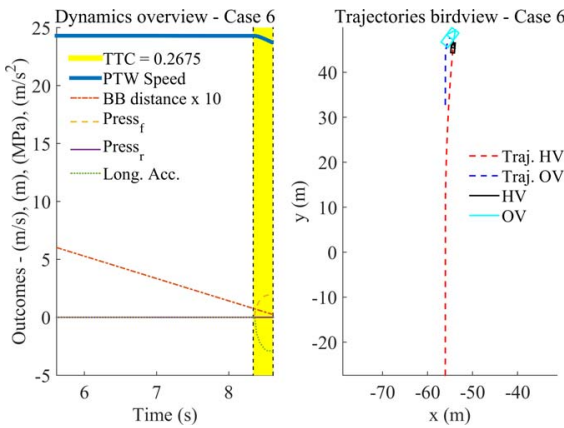

(e)
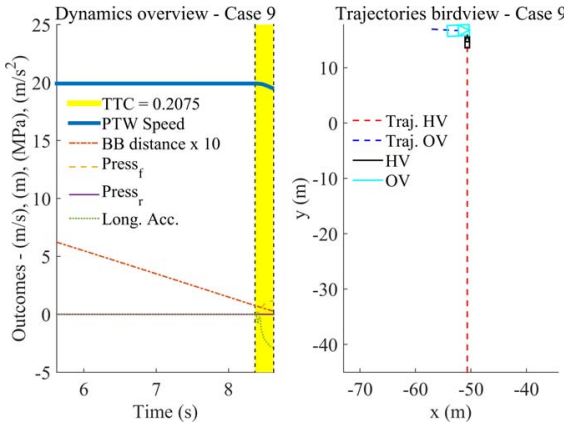

(h)
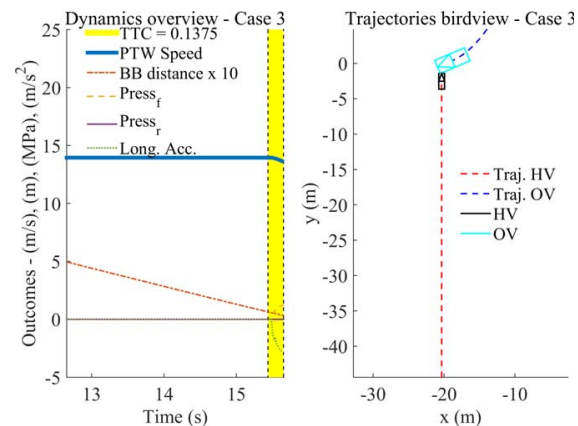

(c)
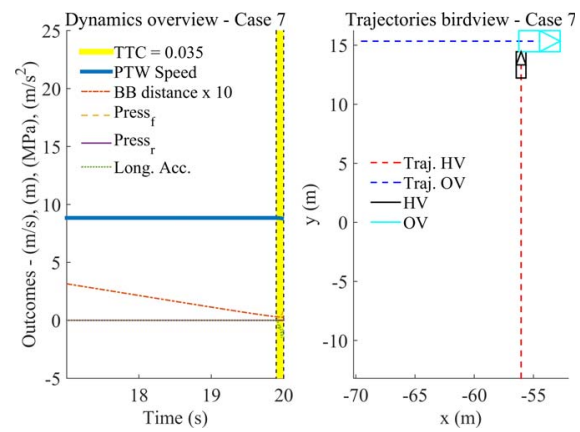

(f)
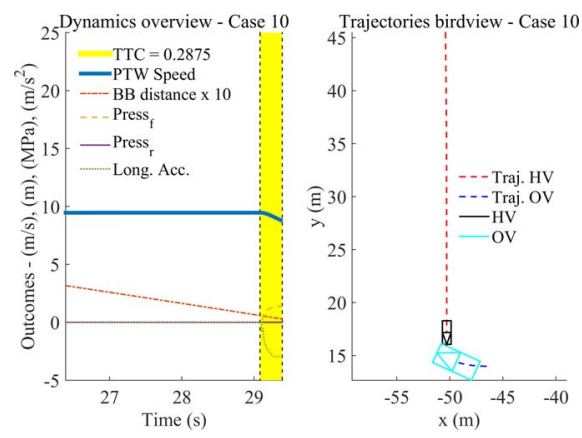

(i)

Fig. 10. Signal plot and trajectory plot of the simulated cases 1 to 3 and 5 to 10 with MAEB intervention. (a) Case 1 . (b) Case 2. (c) Case 3. (d) Case 5. (e) Case 6. (f) Case 7. (g) Case 8. (h) Case 9. (i) Case 10.

\section{CONCLUSION}

This paper presents a method for computing whether a collision between a host motorcycle and an opponent passenger car has become inevitable. This is the first study to apply the inevitable collision state (ICS) construct to single track vehicles, and thus represents an extension of previous work. Experiments simulating real world collision cases confirmed the feasibility of the proposed approach. This approach will support future research on last-resort safety solutions for motorcycle riders, including autonomous emergency braking.

\section{APPENDIX \\ Simulated in-Depth CRAsh Cases}

Case 1: The opponent car approached a give-way line from a side road, intending to turn right into main road. A second vehicle, traveling on main road, stopped and let the opponent car turn. A scooter rider (male, 17) approaching the junction overtook the second vehicle and collided with front left of opponent car. The rider did not attempt any action to avoid the collision

Case 2: The opponent car, at a crossroads, was about to turn right into major road. The driver entered junction and a scooter rider (male, 17), riding on main road, approaching the junction from right (with respect to opponent car), collided with right side of opponent car. The rider did not attempt any action to avoid the collision.

Case 3: The opponent car turned right across two lanes of major road, intending to move from side road to another one. A motorcyclist (male, 35), approaching junction from left (with respect to opponent car), collided with front left of opponent car. The rider did not attempt any action to avoid the collision.

Case 4: The opponent car stopped, intending to cross opposite lane and move into side road. The opponent car was about to turn right when the motorcycle rider (male, 25), overtaking stationary traffic behind the opponent car, collided with the right side of opponent car. The rider did not attempt any action to avoid the collision. 
Case 5: The opponent car turned left into side road. A motorcyclist (male, 43) coming from opposite road hit right front of opponent car. The rider did not attempt any action to avoid the collision.

Case 6: The opponent car coming from side road entered main road. A rider (male, 35) coming from behind tried to overtake opponent car to avoid collision. The opponent car started turning right into a side road thus crossing the motorcycle trajectory. The rider did not attempt any action to avoid the collision. The motorcycle collided with front side part of opponent car.

Case 7: The opponent car was crossing a traffic light controlled junction. A motorcyclist (male, 34) coming from a side road crossed the junction hitting the lateral rear-end part of opponent car. The rider did not attempt any action to avoid the collision.

Case 8: A group of slow moving cars was queuing on approaching a roundabout. The opponent car coming from a side road approached the give-way line intending to turn right in the opposite direction of the queuing cars. A queuing car left a gap to let the opponent car pass. As the vehicle was about to cross the road a scooter rider (male, 16) filtering the queue in the center of the carriageway hit the front right side of the opponent car. The rider did not attempt any action to avoid the collision.

Case 9: The opponent car was about to illegally cross a dual carriageway with a gap connecting the carriageways, turning right from a side road. The motorcyclist approaching from right hand side did not attempt any action to avoid the collision and hit the front of the opponent car.

Case 10: The opponent car approached the give-way line from side road to turn right and come into the main road. A vehicle on the adjacent lane of the main road stopped to let the opponent car turn. A motorcyclist (male, 26) traveling on the center of the carriageway and overtaking the cars in line hit the right front of the opponent car. The rider did not attempt any action to avoid the collision.

\section{ACKNOWLEDGMENT}

The authors would like to thank the project PISa-Powered Two Wheeler Integrated Safety (European Community's Sixth Framework Programme FP6/2000-2006 Grant Agreement 031360) for providing the motorcycle crash cases used in this study.

\section{REFERENCES}

[1] R. A. Blackman and N. L. Haworth, "Comparison of moped, scooter and motorcycle crash risk and crash severity," Accident Anal. Prev., vol. 57, pp. 1-9, Aug. 2013.

[2] E. I. Vlahogianni, G. Yannis, and J. C. Golias, "Overview of critical risk factors in power-two-wheeler safety," Accident Anal. Prev., vol. 49, pp. 12-22, Nov. 2012.

[3] H. Naci, D. Chisholm, and T. D. Baker, "Distribution of road traffic deaths by road user group: A global comparison," Injury Prev., vol. 15, no. 1, pp. 55-59, Feb. 2009.

[4] M. Rizzi, J. Strandroth, A. Kullgren, C. Tingvall, and B. Fildes, "Effectiveness of antilock-brakes (abs) on motorcycles in reducing crashesA multi-national study," in Proc. ESV Conf., 2013, pp. 1-12.

[5] V. Beanland et al., "Acceptability of rider assistive systems for powered two-wheelers," Transp. Res. F, Traffic Psychol. Behav., vol. 19, pp. 63-76, Jul. 2013.
[6] F. Giovannini, G. Savino, M. Pierini, and N. Baldanzini, "Analysis of the minimum swerving distance for the development of a motorcycle autonomous braking system," Accident Anal. Prev., vol. 59, pp. 170-184, Oct. 2013.

[7] G. Savino, F. Giovannini, N. Baldanzini, M. Pierini, and M. Rizzi, "Assessing the potential benefits of the motorcycle autonomous emergency braking using detailed crash reconstructions," Traffic Injury Prev., vol. 14, no. S1, pp. S40-S49, 2013.

[8] T. Fraichard and H. Asama, "Inevitable collision states-A step towards safer robots?" Adv. Robot., vol. 18, no. 10, pp. 1001-1024, 2004.

[9] L. Martinez-Gomez and T. Fraichard, "An efficient and generic 2D inevitable collision state-checker," in Proc. IEEE/RSJ Int. Conf. IROS, 2008, pp. 234-241.

[10] N. Kaempchen, B. Schiele, and K. Dietmayer, "Situation assessment of an autonomous emergency brake for arbitrary vehicle-to-vehicle collision scenarios," IEEE Trans. Intell. Transp. Syst., vol. 10, no. 4, pp. 678-687, Dec. 2009.

[11] A. Eidehall and L. Petersson, "Statistical threat assessment for general road scenes using Monte Carlo sampling," IEEE Trans. Intell. Transp. Syst., vol. 9, no. 1, pp. 137-147, Mar. 2008.

[12] D. Althoff, M. Werling, N. Kaempchen, D. Wollherr, and M. Buss, "Lanebased safety assessment of road scenes using inevitable collision states," in Proc. IEEE IV, 2012, pp. 31-36.

[13] G. Savino et al., "Further development of motorcycle autonomous emergency braking (MAEB), what can in-depth studies tell us? A multinational study," Traffic Injury Prev., vol. 15, no. Sup1, pp. S165-S172, 2014.

[14] M. Althoff and A. Mergel, "Comparison of Markov chain abstraction and Monte Carlo simulation for the safety assessment of autonomous cars," IEEE Trans. Intell. Transp. Syst., vol. 12, no. 4, pp. 1237-1247, Dec. 2011.

[15] V. Cossalter, R. Lot, and F. Maggio, On the Braking Behavior of Motorcycles, SAE Int., Washington, DC, USA, 20044305, 2004.

[16] G. Savino, M. Pierini, and N. Baldanzini, "Decision logic of an active braking system for powered two wheelers," Proc. Inst. Mech. Eng. D, J. Autom. Eng., vol. 226, no. 8, pp. 1026-1036, 2012.

[17] G. Roll, O. Hoffmann, and J. Konig, "Effectiveness evaluation of antilock brake systems (abs) for motorcycles in real-world accident scenarios," in Proc. ESV Conf., 2009, pp. 1-12.

[18] U. Kiencke and L. Nielsen, "Automotive control systems: For engine, driveline, and vehicle," Meas. Sci. Technol., vol. 11, no. 12, p. 1828, 2000.

[19] M. Brannstrom, E. Coelingh, and J. Sjoberg, "Model-based threat assessment for avoiding arbitrary vehicle collisions," IEEE Trans. Intell. Transp. Syst., vol. 11, no. 3, pp. 658-669, Sep. 2010.

[20] B. Fildes et al., "Effectiveness of low speed autonomous emergency braking in real-world rearend crashes," Accident Anal. Prev., vol. 81, pp. 24-29, Aug. 2015.

[21] G. Savino, A. Penumaka, M. Pierini, N. Baldanzini, and B. Roessler, "Design of the decision logic for a ptw integrated safety system," in Proc. ESV Conf., 2009, pp. 1-9.

[22] I. Symeonidis, G. Kavadarli, S. Erich, M. Graw, and S. Peldschus, "Analysis of the stability of ptw riders in autonomous braking scenarios," Accident Anal. Prev., vol. 49, pp. 212-222, Nov. 2012.

[23] G. Savino, M. Pierini, J. Thompson, M. Fitzharris, and M. Lenne, "Exploratory field trial of motorcycle autonomous emergency braking (MAEB): considerations on the acceptability of unexpected automatic decelerations," Traffic Injury Prevent., submitted.

[24] G. Savino, Autonomous Emergency Braking for Powered Two Wheeler Application. Saarbrücken, Germany: LAP, 2013.

[25] O. Gietelink, D. Verburg, K. Labibes, and A. Oostendorp, "Pre-crash system validation with prescan and vehil," in Proc. IEEE Intell. Veh. Symp., 2004, pp. 913-918.

[26] R. Sharp, S. Evangelou, and D. J. Limebeer, "Advances in the modelling of motorcycle dynamics," Multibody Syst. Dyn., vol. 12, no. 3, pp. 251-283, 2004.

[27] G. Savino, M. Pierini, M. Rizzi, and R. Frampton, "Evaluation of an autonomous braking system in real-world ptw crashes," Traffic Injury Prev., vol. 14, no. 5, pp. 532-543, 2013.

[28] VicRoads, VicRoads CrashStats User Guide 2008a, 2008. [Online] Available: http://crashstat1.roads.vic.gov.au/crashstats/appendices.pdf

[29] F. Giovannini, "Studio della manovra evasiva d'emergenza nello sviluppo di un sistema di frenata automatica per veicoli a due ruote," M.S. thesis, Dept. Ind. Eng., Univ. Florence, Florence, Italy, 2010.

[30] G. Savino, J. Brown, M. Rizzi, M. Pierini, and M. Fitzharris, "Triggering algorithm based on inevitable collision states for autonomous emergency braking (aeb) in motorcycle-to-car crashes," in Proc. IEEE Intell. Veh. Symp., 2015, pp. 1195-1200. 


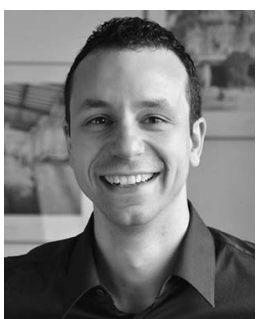

Giovanni Savino received the Master's degree in mechanical engineering and the Ph.D. degree in machine design and construction from University of Florence, Florence, Italy, in 2006 and 2010, respectively.

Since 2005, he has been developing his multidisciplinary expertise in the field of road safety in the Department of Industrial Engineering, University of Florence. He has taken part in several EC-funded projects aimed at improving motorcycle rider safety. These projects include PISa, Saferider, 2BeSafe, and Motorist. In 2012, he was awarded a Research Grant under the EC Marie Curie Actions with his project titled Autonomous BRAking for Motorcycles (ABRAM, Grant 318067), which allowed him to conduct his research at the Monash University Accident Research Center, Australia, during 2013-2015. His main research interests are the development and validation of advanced motorcycle safety technologies.

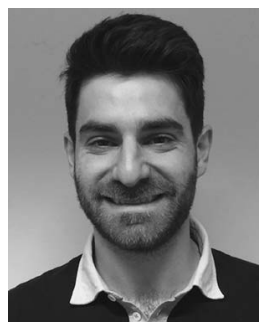

Federico Giovannini was born in Arezzo, Italy. He received the Master's degree in mechanical engineering and the Ph.D. degree in industrial engineering from University of Florence, Florence, Italy, in 2010 and 2014, respectively.

From 2010 to 2014, he worked on motorcycle dynamics and has since been involved in the development of advanced rider assistance systems in the Department of Industrial Engineering, University of Florence. During his Ph.D. studies, he had a sixmonth internship at Yamaha Motorcycle Company in Japan. Since October 2014, he has been a Vehicle Dynamics Engineer with Yamaha Motor Racing, working on the analysis and improvement of motorcycle performance in each race of the MotoGP season.

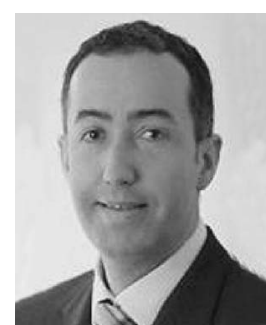

Michael Fitzharris received the B.A. degree in physiology, the B.Sc. degree (first-class honors) in psychology, and the Ph.D. degree in psychology from Monash University, Clayton, Australia, in 1995 and 2006, respectively.

He was the Foundation Director of the Monash University Accident Research Center (MUARC), Johannesburg, South Africa (2008-2010) and is an Associate Director at MUARC, Victoria, Australia, where he heads the In-depth Crash Investigations and Regulation Team. He leads a large in-depth crash investigation research program at MUARC and heads the Enhanced Crash Investigation Study in Victoria.

$\mathrm{He}$ is a specialist in examining the potential benefits of passive and active safety systems and has translated this work within the industry and through global and national regulations.

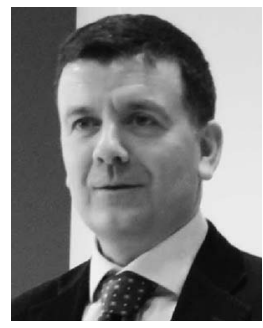

Marco Pierini received the Laurea degree in mechanical engineering from University of Florence, Florence, Italy, in 1991 and the Ph.D. degree in machine design from University of Pisa, Pisa, Italy, in 1995.

In 1994, he was a Guest Researcher with Brel Acoustics, Holte, Denmark. In 1995, he first moved to the Centro Ricerche FIAT (CRF), Turin, Italy; then, he was a Guest Researcher with the Department of Mechanics and Nuclear Engineering, University of Manchester, Manchester, U.K. In 1996, he joined the Department of Mechanical Engineering, Kathoelieke Universiteit Leuven, Leuven, Belgium. In 1998, he returned to University of Florence, where he was first appointed as an Assistant Professor (1998) and then as an Associate Professor (2002). In 2002, he focused his research interest on transport safety, and he was the promoter and founder of the Center for Innovation and Safety of Powered Two Wheelers (CISAP), which he is currently coordinating. 